\title{
Les asynchronismes et les perturbations comme outil d'analyse des problèmes liés au bien-être au travail
}

Kirsti Launis et Juha Pihlaja

\section{(2) OpenEdition}

Journals

Édition électronique

URL : http://journals.openedition.org/activites/1705

DOI : 10.4000/activites. 1705

ISSN : 1765-2723

Éditeur

ARPACT - Association Recherches et Pratiques sur les ACTivités

Référence électronique

Kirsti Launis et Juha Pihlaja, "Les asynchronismes et les perturbations comme outil d'analyse des problèmes liés au bien-être au travail », Activités [En ligne], 4-2 | octobre 2007, mis en ligne le 15 octobre 2007, consulté le 20 avril 2019. URL : http://journals.openedition.org/activites/1705 ; DOI 10.4000/activites. 1705

\section{(c) (i) (9)}

Activités est mis à disposition selon les termes de la licence Creative Commons Attribution - Pas d'Utilisation Commerciale - Pas de Modification 4.0 International. 


\title{
Les asynchronismes et les perturbations comme outil d'analyse des problèmes liés au bien-être au travail.
}

\author{
Kirsti Launis \\ Finnish institute of Occupational Health, Helsinki, Finland \\ Juha Pihlaja \\ Merikoski Rehabilitation and Research Center, Helsinki, Finland
}

\begin{abstract}
Recurrent changes at workplaces seem to increase the haste, stress, health complaints and safety problems of workers. OHS-professionals try to reduce stress or cure separate environmental problems and risk factors at work. However, in spite of their good intentions, in rapid change situations, these professionals may add to the confusion with their prevailing methods and recommendations. The dynamics of workplace transformations are not well understoodfrom theperspective of the everydayworkofeithertheorganization or the individual. In this paper, the Activity Theoretical Approach is used to reconceptualize work-related well-being in situations of rapid change. Key concepts are production and service concepts and their asynchronies. Many asynchronies were observed in the analysis of the two cases of different types of organizations. These asynchronies caused frustration, confusion and lowered the work motivation and perceived well-being of employees. The nature of the load was called disturbance load. Our data and results show that rapid changes in production and service concepts are a major challenge for research on well-being, especially since continuous change has become an inseparable part of work life over the past ten years.
\end{abstract}

KEYWORDS

Activity Theory, production concept, work-related well-being, asynchrony

\section{1.- Introduction}

De nombreuses études ont montré une augmentation du nombre de cas déclarés de stress et de problèmes de santé, notamment depuis le début des années 1990. Une étude très complète (Kompier, Cooper, \& Geurts, 2000) a montré que $25 \%$ des travailleurs européens considéraient que le stress engendrait des problèmes de santé et une moindre performance au travail, tout en reconnaissant que leurs conditions de travail avaient été améliorées et que l'offre en services de santé au travail ainsi qu'en formations professionnelles avait augmenté (European Agency fo Safety and Health at Work, 2002; De Greef, \& Van den Broek, 2004). Pour Doherty, Forslin, Shani et Kira (2002), l'intensité du travail a augmenté depuis le début des années 1990 car la gestion des firmes est de plus en plus guidée par des objectifs de compétitivité à court terme. Si de plus en plus d'aspects de la vie professionnelle semblent source de problèmes pour le personnel, faut-il considérer pour autant que le management en est le seul responsable?

Pour faire face à ces problèmes, les entreprises mobilisent les compétences de professionnels de la santé, de la sécurité, de l'ergonomie et du développement personnel. En dépit de leurs bonnes intentions, ces professionnels peuvent ajouter une part de confusion dans les situations à évolution rapide, 
Les asynchronismes et les perturbations comme outil

d'analyse des problèmes liés au bien-être au travail

en mobilisant leurs concepts usuels de prévention en matière de santé et de sécurité, et en utilisant les modélisations du travail et les outils qu'ils mobilisent habituellement. Cependant, tout en cherchant à réduire le stress ou les facteurs de risque au travail pour les individus, ou à traiter les divers problèmes de l'environnement de travail en utilisant des modèles traditionnels, ils peuvent au contraire aider au maintien des problèmes associés aux transformations rapides des situations de travail. Nous considérons qu'une part des problèmes inhérents au bien-être professionnel s'ancre dans l'usage des théories, modèles et outils des experts. Par exemple, la théorie dominante au sein des services de santé au travail est celle de l'épidémiologie. La théorie de l'épidémiologie, et les outils développés à partir de cette dernière, n'est pas suffisante pour comprendre les problèmes liés au bien-être engendrés par les processus de transition au sein des situations de travail.

De manière à survivre dans un milieu toujours plus compétitif, les entreprises cherchent constamment à mettre en œuvre de nouveaux concepts pour rendre plus fonctionnelles et plus productives les activités de production et de services. Même si la littérature est abondante au sujet de ces changements continuels, il n'est pas facile d'identifier le contexte et les dynamiques inhérentes à ces événements, qui ne sont bien compris ni par l'entreprise ni par les individus. Lorsque les activités de production et de service existants au sein de l'entreprise ne correspondent plus aux exigences du marché, ou lorsque de nouveaux concepts sont lancés pour réaliser ces activités, plusieurs perturbations se produisent au sein de l'entreprise (Engeström, 1988; Mäkitalo, 2005). Mais, le processus de transition n'est pas si fluide et linéaire que ce qu'annoncent les managers, les experts et les consultants. Les équipes de travail et les travailleurs isolés vivent souvent les changements comme un challenge, mais les trouvent également éprouvants. La continuité du travail quotidien est entravée par des interruptions, des perturbations, des accélérations, ou par des problèmes qui sont souvent individualisés comme le défection de certaines catégories de travailleurs (personnes âgées, nouveaux arrivants, managers, planificateurs), et des essais sont faits afin de faire face à ces difficultés par la formation ou par la création de nouvelles procédures. C'est pourquoi, dans nos interventions, nous avons commencé par étudier le processus de transition lui-même plutôt que des caractéristiques propres de travailleurs individuels ou que des facteurs spécifiques de stress au travail.

\section{2.- Question de recherche, méthodes et données}

Nous avons étudié les processus de changement de plusieurs organisations du travail finlandaises pendant deux années (2002-2004) au cours desquelles nous sommes intervenues dans ces entreprises en nous basant sur la théorie culturelle et historique de l'activité (Engeström, Lompscher, \& Ruckriem 2005) et la méthode du " Change Laboratory » (Virkkunen, Engeström, Helle, Pihlaja, \& Poikela, 1997). La théorie culturelle et historique de l'activité nous a permis d'énoncer les questions de recherche que nous retenons pour cet article. Les données présentées sont issues des interventions menées selon la méthode du " Change Laboratory ». Cependant, les processus d'intervention ne sont pas décrits dans ce qui suit.

\section{1.-Questions de recherche}

Bien que les problèmes de bien-être psychosocial au travail soient basés sur des expériences individuelles, plutôt que de se focaliser sur les individus, nous avons préféré nous focaliser sur les transitions au sein des processus de travail. Notre hypothèse était que le bien-être professionnel dépendait étroitement de la réussite professionnelle, et que les problèmes de bien-être provenaient de situations pour lesquelles la continuité du processus de travail était perturbée. En outre, dans ces processus de transition, le nombre de ces situations perturbatrices, de « bruit dans le flux de travail » (Engeström, 1988) augmentent notablement. Nous avons recueilli les données sur les situations perturbatrices au cours de nos interventions, et nous souhaitons traiter dans l'article les questions suivantes: (i) quels types de processus de changement se produisent au sein de l'organisation? (ii) quels types de 
Les asynchronismes et les perturbations comme outil

d'analyse des problèmes liés au bien-être au travail

perturbations peuvent résulter de ces changements? (iii) quelle est la relation entre le changement et le bien-être professionnel? Dans cet article, nous montrons que le bien-être est lié non seulement aux changements quantitatifs de la charge de travail, mais également aux changements qualitatifs et historiques de la production.

\section{2.-La théorie culturelle et historique de l'activité}

$\mathrm{Au}$ lieu de choisir comme unité d'analyse les travailleurs individuels ou des configurations de facteurs spécifiques tant environnementaux que de stress, nous avons choisi les situations locales de changement, en intégrant dans l'analyse les dynamiques d'évolution du système d'activité propres aux organisations étudiées. Le développement historique, et les changements continuels du système de l'activité (Engeström, 1987; 2000), ont servi de contour à notre travail mais n'ont en rien restreint notre analyse et nos interprétations.

Nous avons conceptualisé les changements des systèmes d'activité comme des changements historiques et qualitatifs apparus au niveau des concepts associés à l'activité de production en utilisant les types d'organisation du travail historiquement constitués tels que décrits par Victor et Boynton (1998). Ces types d'organisation du travail représentent les différents principes selon lesquels la production (ou les services) est organisée. Afin de mettre en oeuvre de telles transformations d'un type à un autre, les entreprises doivent découvrir de nouvelles manières d'organiser le travail, de nouveaux procédés, de nouveaux types de connaissances, de nouvelles relations entre les managers et les travailleurs, et de nouvelles formes de systèmes d'information. Nous avons développé un modèle descriptif (Launis, \& Pihlaja, 2005 ; Pihlaja, 2005) pour réaliser l'analyse théorique des changements survenus dans les concepts orientant l'activité de production et de services. Dans la matrice historique, nous décrivons ainsi de manière détaillée quatre concepts de production et de services: 1) la production artisanale, 2) la production de masse, 3) la production de masse flexible et 4) la production de masse ajustée sur mesure.

\section{3.-Nos données}

Nos données proviennent de deux types d'organisations: une fabrique de papier, organisée comme une organisation industrielle traditionnelle, et une entreprise municipale, qui fournit des services de ménage et de restauration ainsi que d'entretien de bâtiments. Nous avons choisi ces deux exemples parce que le premier représente une organisation privée et industrielle tandis que le second est une organisation publique de services. Nous avons utilisé les données issues de ces cas comme des exemples illustrant des situations et non pour instruire une comparaison entre ces deux organisations.

Lors de nos interventions, notre principal objectif était de promouvoir les processus de transition dans les organisations, notamment dans des unités locales, en coopération avec le personnel. Avec ce dernier et les spécialistes de la santé et de la sécurité, nous avons analysé les processus de changement dans les organisations. Nos données sont constituées à partir de différents matériaux rassemblés et collectés au cours de nos interventions dans les unités de travail pilotes de l'organisation. Les données sont constituées: 1) des descriptions des perturbations et des situations perçues comme désagréables par le personnel dans les unités de travail, 2) des documents faisant état de la stratégie, de la politique et des projets de l'entreprise, 3) des entretiens avec le personnel et des managers, 4) des documents relatifs aux solutions et aux décisions prises lors des situations de changement d'organisation, 5) des enregistrements et des documents retraçant le développement du processus au cours de l'intervention, et 6) de nos propres observations au sein des entreprises.

\section{3.- Résultats et discussion}

Nos résultats ont montré que les concepts orientant la production ou les services ont changé rapide- 
Les asynchronismes et les perturbations comme outil

d'analyse des problèmes liés au bien-être au travail

ment dans les organisations étudiées après la fin des années 1990. Jusqu'au début des années 1990, le concept de production de masse ou son équivalent pour les services était prédominant mais, à la fin de la décennie, de nouveaux concepts sont apparus et l'évolution s'est faite toujours plus rapidement. Le développement de ces nouveaux concepts de production est de fait devenu essentiel pour survivre au sein d'un marché compétitif. Mais, lorsque les processus de changement étaient conduits au plus près du terrain, beaucoup de perturbations, d'asynchronismes et d'échecs ont été observés dans les organisations, que ce soit dans les dimensions verticale ou horizontale de l'organisation du travail.

\section{1.-Le cycle croissant des changements}

Dans l'entreprise industrielle, nous avons constaté un rythme rapide de passage d'un concept à un autre (tableau 1) : après un siècle de production de masse, en 1993, l'entreprise a sombré dans une crise profonde parce que les exportations vers l'union soviétique (un tiers de sa capacité totale) furent coupées du jour au lendemain. Il y eut des fermetures d'usines, des menaces de licenciement, l'atmosphère était alors oppressante. Afin de survivre à cette compétition, la qualité des produits, la fiabilité, et l'efficience des coûts devaient être améliorées de manière substantielle. En collaboration avec le personnel - en recourant à des modèles de participation et de travail d'équipe - les managers ont développé un nouveau concept de production - la production de masse flexible. En 1995, un nouveau système de qualité fut élaboré et de nouvelles solutions de traitement de l'information ont été introduites. À la fin des années 1990, des choix stratégiques ont été faits pour sélectionner les clients stratégiques et mettre en place une production tournée vers le client.

\begin{tabular}{|c|c|c|c|}
\hline Années & $\begin{array}{c}\text { Production de } \\
\text { masse } \\
\text { standardisée }\end{array}$ & $\begin{array}{c}\text { Production de } \\
\text { masse flexible }\end{array}$ & $\begin{array}{c}\text { Production de } \\
\text { masse sur } \\
\text { mesure }\end{array}$ \\
\hline$\ldots$ & & & \\
\hline 1991 & & & \\
\hline 1992 & & & \\
\hline 1995 & & & \\
\hline 1996 & & & \\
\hline 1999 & & & \\
\hline$\ldots$ & & & \\
\hline
\end{tabular}

Tableau 1: Changements des concepts de production dans l'entreprise industrielle au cours des dix dernières années.

\section{2.-Les perturbations actuelles révèlent la superposition de multiples concepts}

Les résultats du tableau 1 se basent sur les entretiens et les informations récoltées (pendant les interventions). Selon les données recueillies pendant les entretiens, les processus de changement semblent avoir été mis en œuvre sans accroc, mais la description des perturbations quotidiennes a remis en cause cette image idyllique. Lorsque nous avons demandé aux travailleurs de la papeterie de décrire des situations concrètes qu'ils considéraient comme éprouvantes, presque toutes les descriptions faites étaient relatives au système de gestion des données mis en place récemment pour piloter la production.

Après le projet de développement, nous avons analysé: 1) Quel concept de production sous-tendait ce système de données, 2) Quel type de formation était en place lorsque le système de gestion des données a été lancé, 3) Quelles solutions les travailleurs ont proposés lorsqu'ils décrivaient leurs difficultés, 4) Quelles solutions ont été développées lorsque la formation s'est révélée insuffisante, 5) Quelles solutions les travailleurs ont développé au-delà des limites organisationnelles traditionnelles. Les résultats sont synthétisés au sein du tableau 2.

Le système de gestion des données a été élaboré pour permettre à l'ensemble de l'entreprise de dé- 
Les asynchronismes et les perturbations comme outil

d'analyse des problèmes liés au bien-être au travail

velopper un système de production sur mesure et adapté (1) (les numéros se rapportent aux lignes du tableau 2). La formation organisée pour mettre en œuvre le nouveau système de gestion des données se basait sur le concept de production de masse: ainsi chaque travailleur était formé selon la division du travail existante, dans la sphère de ses propres missions (2). Lors de la mise en œuvre du nouveau système, les travailleurs durent faire face à de nombreux problèmes, et, pour résoudre ces derniers, ils proposèrent que la division du travail et que les responsabilités individuelles soient précisées et formalisées de manière claire, ce qui renvoyait de fait au concept précédent de production de masse (3). Au cours de l'intervention, le personnel mit en place des groupes de travail au sein des unités de travail de manière à organiser des forums de discussion réguliers, permettant d'analyser et de résoudre les problèmes lorsqu'ils se produisaient (ce qui renvoie à l'idée d'amélioration continue) (4). Plus tard, il fut également admis qu'une utilisation efficace et appropriée du système nécessitait la tenue de réunions régulières transcendant les limites des différents secteurs, de manière à résoudre les problèmes et à développer et utiliser le système dans une démarche tournée vers le client (5).

\begin{tabular}{|c|c|c|c|}
\cline { 2 - 4 } \multicolumn{1}{c|}{} & $\begin{array}{c}\text { Productioe de } \\
\text { masse } \\
\text { staedardisée }\end{array}$ & $\begin{array}{c}\text { Productioe de } \\
\text { masse flexible }\end{array}$ & $\begin{array}{c}\text { Productioe de } \\
\text { masse sur } \\
\text { mesure }\end{array}$ \\
\hline $\begin{array}{c}\text { (1) Système de } \\
\text { gestioe de la } \\
\text { productioe }\end{array}$ & & & \\
\hline $\begin{array}{c}\text { (2) Système de } \\
\text { formatioe }\end{array}$ & & & \\
\hline $\begin{array}{c}\text { (3) Solutioes } \\
\text { proposées }\end{array}$ & & & \\
\hline $\begin{array}{c}\text { (4) Solutioes } \\
\text { développées }\end{array}$ & & & \\
\hline (5) Solutioes \\
développées
\end{tabular}

Tableau 2: Asynchronismes lors des transitions au sein d'une organisation industrielle.

Avant l'intervention, les problèmes identifiés étaient souvent formulés comme le fruit d'une limite dans les compétences et le savoir-faire des employés. Nous appelons asynchronismes les situations dans lesquelles les diverses fonctions hiérarchiques et les divers secteurs de l'organisation essaient de mettre en œuvre des concepts de production différents. Les asynchronismes horizontaux que nous avons décrits ont été partiellement surmontés par la mise en œuvre de solutions par lesquelles les frontières entre les différents secteurs à la base de l'organisation ont été dépassées, et que les nouvelles demandes des clients ont été abordées de manière concertée.

\section{3.-Évoluer entre les concepts - éprouvant et frustrant}

En 2002, un des objectifs des managers de l'organisation de service municipal était de développer un travail d'équipe en autogestion au sein des équipes locales de services. L'idée était que les travailleurs affectés aux trois types de poste différents (nettoyage, entretien des bâtiments, restauration), pouvaient, de leur propre initiative, s'entraider, au-delà des frontières traditionnelles entre les corps de métier. Nous avons interprété cet objectif comme étant une tentative de transition d'un concept de production de masse vers la fourniture d'un service flexible (1), (les numéros se réfèrent aux lignes du tableau 3). Cependant, les travailleurs de ces équipes pilotes n'étaient pas intéressés par ce modèle de travail d'équipe. Au contraire, ils voulaient revenir à l'ancienne division du travail basée sur des instructions standardisées (2). Néanmoins, dans ce contexte classique de division du travail, il se produisait des situations dans lesquelles tous les travailleurs du groupe s'entraidaient. Cependant, ces dépassements des limites entre corps professionnels étaient temporaires et les travailleurs retournaient aussitôt à leurs propres tâches, telles que décrites dans leurs fiches de poste (3). Au cours de l'intervention, les travailleurs élaborèrent, avec les chercheurs et les spécialistes des services de 
Les asynchronismes et les perturbations comme outil d'analyse des problèmes liés au bien-être au travail

santé et sécurité au travail, un nouveau modèle de production, basé sur l'idée d'un partenariat, que nous avons considéré comme un modèle de production sur mesure. Ce modèle, pour être mis en place, devait d'abord être accepté par les managers, mais s'inscrivait dans la ligne de la politique de la direction en 2003 (4). Cependant, la direction du centre décida, en 2004, de réorganiser les services en trois lignes de production indépendantes: la restauration, l'entretien des bâtiments, et le nettoyage (5). Cette organisation en ligne signifiait un retour à une organisation classique de services de masse, et créa une frustration parmi les membres des équipes: " c'est mieux si on fait juste notre travail quotidien. Je savais déjà dès le début du projet qu'ils ne voudraient pas appliquer les idées des travailleurs » (un membre d'équipe, 2003).

Nous appelons asynchronismes dans les processus de changement ces allers et retours et revirements. Les asynchronismes décrits ici sont verticaux (entre la direction et les travailleurs à la base), et semblent avoir été la cause principale des problèmes évoqués au sein du personnel concernant leur bienêtre professionnel. Nous pouvons, cependant, nous demander si les modèles développés pendant l'intervention étaient de fait réalistes. Est-ce que les idées des travailleurs, et la vision de la direction, n'étaient pas trop éloignées des concepts classiques et dominants de la production de masse? Existait-il un écart insurmontable entre les objectifs identifiés et les pratiques existantes?

\begin{tabular}{|c|c|c|c|}
\cline { 2 - 4 } \multicolumn{1}{c|}{} & $\begin{array}{c}\text { Service de } \\
\text { masse } \\
\text { standardisé }\end{array}$ & $\begin{array}{c}\text { Service de } \\
\text { masse exible }\end{array}$ & $\begin{array}{c}\text { Service de } \\
\text { masse sur } \\
\text { mesure }\end{array}$ \\
\hline $\begin{array}{c}\text { (1) Direction : soutien des } \\
\text { équipes }\end{array}$ & & & \\
\hline $\begin{array}{c}\text { (2) Unité de service } \\
\text { spécialisation professionnelle }\end{array}$ & & & \\
\hline $\begin{array}{c}\text { (3) Aller au-delà des limites } \\
\text { professionnelles classiques et } \\
\text { revenir }\end{array}$ & & $\longrightarrow-$ - & \\
\hline $\begin{array}{c}\text { (4) Nouveau modèle de service } \\
\text { élaboré par les travailleurs }\end{array}$ & & & \\
\hline (5) Re-organisation & & & \\
\hline
\end{tabular}

Tableau 3: Les asynchronismes dans la transition d'une organisation de service

\section{4.- Conclusion}

Les changements divergents et de plus en plus rapides dans les concepts de production et de services posent des problèmes à la fois en terme de continuité de l'activité et de bien-être professionnel. Lorsque les solutions sont recherchées pour favoriser le bien-être professionnel, elles sont trouvées dans les processus de transition des concepts de production. Cela ne signifie pas que nous devons accepter les concepts et modèles dominants de gestion et de management, mais que, de manière préventive, il est nécessaire d'analyser et de conceptualiser les problèmes relatifs au bien-être professionnel dans le contexte d'évolution de ces concepts. Promouvoir le bien-être professionnel nécessite la mise en œuvre de nouvelles approches théoriques: la recherche et les méthodes d'analyse doivent permettre d'être capable de relier l'ensemble de l'organisation et les perturbations perçues par les travailleurs, plutôt que de les séparer en plusieurs (micro-macro) niveaux. La théorie culturelle et historique de l'activité fournit de bonnes bases pour cela.

Nos données et nos résultats montrent que les changements dans les concepts de production et de service représentent un enjeu important pour la recherche portant sur le bien-être, notamment parce que les changements et les évolutions continuelles sont devenus incontournables au sein de nos vies 
professionnelles modernes depuis le début des années 1990.

Afin de traiter la redéfinition de la production/ des services sans engendrer de nouveaux problèmes de santé ou de sécurité pour les employés, de nouvelles formes de rencontres et d'alliances sont nécessaires entre les directions, les concepteurs du bureau des méthodes, les spécialistes de la santé et de la sécurité, et les communautés locales de travail. Un langage commun n'est pas le seul élément requis par cette nouvelle forme de collaboration. Des conceptualisations, modèles et outils novateurs sont nécessaires afin d'analyser et d'interpréter les processus de transition, et de résoudre les asynchronismes émergents et les échecs dans les concepts de production et de services. Lors de processus sévères de transformation, la bonne marche des processus de production et de services améliore à la fois la santé et la sécurité du personnel, ainsi que la productivité.

\section{RÉFÉRENCEMENT}

Launis, K., \& Pihlaja, J. (2007). Les asynchronismes et les perturbations comme outil d'analyse des problèmes liés au bien-être au travail. @ctivités, 4(2) pp. 91-98, http://www.activites.org/v4n2/v4n2. pdf

\section{BiBLIOGRAPHIE}

De Greef, M., \& Van den Broek, K. (2004). Quality of the working environment and productivity. Research findings and case studies, European Agency for Safety and Health at Work.

Docherty, P., Forslin, J., Shani, A., \& Kira, M. (2002). Emerging work systems: from intensive to sustainable. In P. Docherty, J. Forslin, \& A. Shani (Eds.), Creating Sustainable Work Systems. Emerging Perspectives and Practice. London: Routledge.

Engeström, Y. (1987). Learning by Expanding. Jyväskylä: Gummerus Oy.

Engeström, Y. (1988). Reconstructing work as an object of research. The Quarterly Newsletter of the Laboratory of Comparative Human Cognition, 10 (1), 21-27.

Engeström, Y. (2000). Activity theory as a framework for analyzing and redesigning work. Ergonomics, 34 (7), 960-974.

Engeström, Y., Lompscher, J., \& Ruckriem, G. (Eds.) (2005). Putting Activity Theory to Work. Contributions from Developmental Work Research, ICHS, Volume 13. Berlin: Lehmanns Media.

European Agency for Safety and Health at Work. (2002). Research on changing world of work.

Kompier, M., Cooper, C., \& Geurts, S. (2000). A multiple case study approach to work stress prevention in Europe. European Journal of Work and Organizational Psychology, 9 (3), 371-400.

Launis, K., \& Pihlaja, J. (2005). Production concepts and learning: Changes and work-related well-being. Paper presented in RWL-conference in Sydney, December.

Mäkitalo, J. (2005). Work-related well-being in the trans-formation of nursing home work. University of Oulu, D 837. Oulu.

Pihlaja, J. (2005). Learning in and for Production. An Activity Theoretical Study of the Historical Development of Distributed Systems of Generalizing. Helsinki: University Press.

Victor, B., \& Boynton, A. (1998). Invented here. Maximizing Your Organization's Internal Growth and Profitability. Boston: Harvard Business School Press.

Virkkunen, J., Engeström, Y., Helle, M., Pihlaja, J., \& Poikela, R. (1997). The change laboratory - a tool for transforming work. In T. Alasoini, M. Kyllönen, \& A. Kasvio (Eds.), Workplace Innovations - A Way of Promoting Competitiveness, Welfare and Employment. Helsinki: National workplace development programme/Ministry of labor. 


\section{RÉSUMÉ}

Il semblerait que les changements récurrents au sein des situations de travail tendent à augmenter les plaintes liées au stress, aux cadences, à la santé ainsi que le nombre des problèmes liés à la sécurité des travailleurs. Les professionnels de la santé et de la sécurité du travail cherchent à réduire ce stress et à traiter divers facteurs de risque ou divers problèmes liés à l'environnement de travail. Cependant, malgré leurs bonnes intentions, ces professionnels peuvent aussi ajouter une part de confusion avec leurs méthodes préventives et leurs recommandations faites au sein de situations marquées par la rapidité des changements qui s'y produisent. Les dynamiques de transformations des milieux professionnels ne sont pas bien comprises et appréhendées à partir d'un regard sur le travail quotidien, que ce soit du point de vue de l'organisation toute entière ou de celui de l'individu. Cet article propose d'utiliser l'approche théorique de l'activité pour conceptualiser autrement le bien-être professionnel dans les situations de changement rapide. Les concepts clés mobilisés sont les concepts qui orientent l'activité de production et de services, ainsi que de leurs asynchronismes. De multiples asynchronismes ont été observés au sein des analyses qui nous avons menées dans deux entreprises. Ces asynchronismes ont occasionné de la frustration et de la confusion, et ont diminué le niveau de motivation au travail et la perception de bien-être des employés. La nature de la charge correspondante a été dénommée la « charge de perturbation ». Les données et les résultats que nous avons obtenus montrent que les changements rapides des concepts sous-jacents aux activités de production et de services constituent un enjeu majeur pour la recherche sur le bien-être, notamment puisque les changements continuels au cours d'une carrière professionnelle sont désormais devenus incontournables depuis ces dix dernières années.

\section{MotS CLÉS}

théorie de l'activité, concept de production, bien-être professionnel, asynchronisme

\section{RESUMEN}

Los cambios constantes en los lugares de trabajo parecen incrementar el apuro, el estrés, las quejas sobre la salud y los problemas de seguridad de los trabajadores. Los profesionales de la salud y de la seguridad laboral intentan reducir el estrés o remediar diferentes problemas de medio ambiente y factores de riesgo en el trabajo. Sin embargo, a pesar de sus buenas intenciones, en situaciones de cambios rápidos, estos profesionales pueden generar confusión con sus métodos y recomendaciones habituales. No comprenden bien la dinámica de las transformaciones del puesto de trabajo desde la perspectiva del trabajo diario tanto de la organización como de los individuos. En este artículo, utilizamos el enfoque teórico de la actividad para reconceptualizar el bienestar atribuible al trabajo en situaciones de cambios rápidos. Los conceptos clave son los de producción y servicio, así como el de sus asincronías. Se observaron varias asincronías en el análisis de los dos casos en diferentes tipos de organizaciones. Estas asincronías causaron frustración, confusión y disminuyeron la motivación laboral y la sensación de bienestar de los empleados. El tipo de carga se dio a llamar carga de perturbación. Nuestros datos y resultados muestran que los cambios rápidos en los conceptos de producción y de servicio son un desafío importante para la investigación sobre el bienestar, especialmente desde que los cambios continuos se han transformado en una parte inseparable de la vida laboral desde los últimos diez años.

\section{Palabras ClaVes}

Teoría de la actividad, concepto de producción, bienestar asociado al trabajo, asincronía 